\title{
Simulation-based Scheduling of a Large-scale Industrial Formulation Plant Using a Heuristics-assisted Genetic Algorithm
}

\author{
Christian Klanke \\ christian.klanke@tu-dortmund.de \\ TU Dortmund University \\ Dortmund, Germany
}

\author{
Dominik Bleidorn \\ dominik.bleidorn@inosim.com \\ INOSIM Software GmbH \\ Dortmund, Germany
}

\author{
Christian Koslowski \\ christian.koslowski@inosim.com \\ INOSIM Software GmbH \\ Dortmund, Germany
}

\author{
Christian Sonntag \\ christian.sonntag@inosim.com \\ INOSIM Software GmbH \\ Dortmund, Germany
}

\author{
Sebastian Engell \\ sebastian.engell@tu-dortmund.de \\ TU Dortmund University \\ Dortmund, Germany
}

\begin{abstract}
Research has brought forth several promising approaches, e.g. Mixedinteger linear programming [8] or Constraint Programming [13] to represent industrial batch production plants and to optimize their production schedules. But still, after decades of research in the field, scheduling is done (semi)-manually in industry in almost all cases. Main reasons for this besides the intrinsic combinatorial complexity are that model development and maintenance require expert knowledge. This work tackles these challenges by using a simulation-based optimization approach in which a Genetic Algorithm provides high-level encodings of schedules and an industrialstrength discrete-event simulator that provides a detailed model of the plant and is used as a fitness evaluator. To enable this approach to solve problems of industrial complexity, the scheduling heuristics are used to reduce the search space to a reasonable size that still contains the important degrees of freedom so that still optimal or at least high-quality solution are obtained. The case study that is considered here is an industrial two-stage formulation plant which is modeled in detail, down to the level of shiftmodels of the operators and mass-balances from source to sink, thus ensuring that the computed schedules are directly applicable at the real-world plant.
\end{abstract}

\section{CCS CONCEPTS}

- Applied computing $\rightarrow$ Supply chain management.

\section{KEYWORDS}

Industrial production scheduling, Genetic Algorithm, Discrete-event simulation, Heuristics

ACM Reference Format:

Christian Klanke, Dominik Bleidorn, Christian Koslowski, Christian Sonntag, and Sebastian Engell. 2021. Simulation-based Scheduling of a Largescale Industrial Formulation Plant Using a Heuristics-assisted Genetic Algorithm. In 2021 Genetic and Evolutionary Computation Conference Companion

\section{(c) (†) \$}

This work is licensed under a Creative Commons Attribution-NonCommercial International 4.0 License.

GECCO '21 Companion, July 10-14, 2021, Lille, France

(C) 2021 Copyright held by the owner/author(s).

ACM ISBN 978-1-4503-8351-6/21/07.

https://doi.org/10.1145/3449726.3463176
(GECCO '21 Companion), July 10-14, 2021, Lille, France. ACM, New York, NY, USA, 9 pages. https://doi.org/10.1145/3449726.3463176

\section{INTRODUCTION}

Improvements in the scheduling of industrial processing plants can lead to significant improvements of their performance without investments in new equipment. However, building a scheduling system for frequent use is challenging. A key requirement is to consider all relevant features and constraints of the production plant accurately so that the computed production schedules are applicable to the real plant without further intervention.

Additional requirements are imposed by the need for user acceptance long-term maintainability of such a system in an industrial environment. These include the ability to easily make changes, e.g. to account for changing product portfolios, and to make the system easy to be used by the personnel in the plant. Exact solution approaches such as mixed-integer linear programming or constraint programming are problematic with respect to these requirements. Usually they lack the ability to represent all features of processing plants at the level of detail that production planners require for industrial applications. The expert knowledge that is required for the setup and maintenance of such solutions is significant and increases the dependency on highly trained personnel.

Because of these requirements, in this work a simulation optimization approach is proposed that combines a Genetic Algorithm (GA) with a discrete-event simulator (DES). A DES treats the plant operations as a sequence of events in time, where each event marks a change in the state of the system. Between two consecutive events, no state-transitions are assumed to take place. We here use the INOSIM Software, which is an industry-proven software for daily use with adequate of modeling capabilities and comfort of use. It provides the possibility of accurately representing highly complex industrial processing plants. The software can also generate schedules by applying scheduling rules, but due to the local nature of these decisions, the schedules generated by DES are usually suboptimal. We therefore combine it with a GA that performs a search for good schedules in the global solution space instead of applying local rules.

The search space of industrial scheduling problems is usually huge, rendering problems of realistic size computationally intractable if all decisions are included. Therefore, in order to obtain highquality solutions within a reasonable amount of time, a reduction 
of the search-space is needed. In our approach, the GA only encodes those degrees of freedom, that have a high impact on the solution quality, while the remaining degrees of freedom are dealt with in a heuristic manner either on the level of the encoding in the GA or directly by the DES at simulation time.

Similar approaches have already been developed for a variety of problem settings. In [11] the operation of a pipeless plant that uses automated guided vehicles to transport substances between processing stations was optimized with respect to the makespan of the process. The approach encodes production sequence of the batches that are processed. This production sequence is implemented such that precedence constraints between subsequent operations of a job are satisfied automatically. The resulting schedules are evaluated by simulation of the pipeless plant which serves as a schedule builder and applies decision rules. Repair algorithms and penalties for infeasibility are employed to deal with the remaining constraints.

In [5] the Technomatix Plant Simulation software was used to optimize the manufacturing of steel pipes for the oil and gas industry, using a cost objective that includes changeover, inventory and penalty costs, using the integrated GA provided by the software. The sequence as well as the release times of the batches were varied.

In [6] a reentrant hybrid flow shop problem in a multi-objective problem setting was solved. Among others, the non-dominated sorting genetic algorithm NSGA-II is used to coordinate solutions that are evaluated by a DES in a stochastic manner. In the encoding, each gene in the chromosome represents a policy that manages the queue on a machine during the simulation. The authors compare their optimization approach with the best results obtained by full enumeration of all solutions and conclude that their approach yields solutions very close to the Pareto-optimum.

In [10] NSGA-II in combination with the Witness Simulation Software was used to optimize the frequencies for preventive maintenance in a multi-objective setting. The optimization variables are the total costs due to maintenance and the profit from product sales. The case study is a two-stage flow shop with limited intermediate storage, consisting of five identical units in the first stage, and a different unit in the second stage. Each type of unit has three maintenance operations associated with them, for a total of six operations. The maintenance frequency for each of these six operations is optimized by the evolutionary algorithm. The results show a significant improvement over the unoptimized case and provide insights into the individual contribution of the maintenance operations. One major caveat is the optimization time of 383 hours, which makes this approach only feasible for industrial applications where the problem has to be solved only occasionally.

In [9], the batching and batch scheduling problem in a hybrid flow shop was addressed, with the goal of minimizing production costs without violating delivery due dates. The examined model is based on a real-world manufacturing plant and consists of five stages, with a total of 11 machines. The simulation is conducted using the Tecnomatrix Plant Simulation Software, while the optimization is done via a genetic algorithm. Individuals consist of two genes per product, one for the batch size and one for the batch entry probability. First all batch size genes are encoded, followed by batch sequence genes. Within the simulation, tasks are scheduled via the First-In-First-Out heuristic. With this approach, the authors reach the optimal solution for the total cost, without violating due dates, in 59 minutes of total computation time.

Overall, simulation optimization approaches are promising to solve industrial scheduling problems which are intractable for exact solution methods. The examples discussed above show that by reducing the problem to a smaller subset of decision variables it is possible to achieve near-optimal solutions with reasonable computation times.

We follow this general idea when modeling and optimizing the operation of a real-world two-stage industrial batch plant, that is operated by a large provider of special chemicals. Currently, the scheduling of the production plant is done manually and our proposed approach aims at automating this procedure, while improving the quality of the schedules at the same time. The remainder of this paper is structured as follows: At first a detailed description of the industrial plant and the DES model is given in Section 2. Then the optimization approach is presented in Section 3. The focus is on the encoding of the schedules in the GA, the heuristics to reduce the size of the search-space, and the interaction between GA and DES. In Section 4, the results obtained with this approach are presented, the performance is compared to simpler approaches and the reproducibility of the results is discussed. We conclude our contribution in Section 5 and give an outlook on future research directions.

\section{CASE STUDY}

The industrial processing plant investigated in this contribution is shown in Figure 1. The plant consists of two stages, the formulation and the filling stage. A transfer panel connects the two stages with each other and with intermediate buffer tanks.

The formulation and the filling stage are decoupled by buffer tanks, to increase the overall productivity of the plant. To free up production capacities in the formulation lines after intermediates have been produced, the intermediates are usually routed through the buffer tanks instead of charging the filling stations directly. Charging the buffer tanks with the intermediates from the formulation lines is fast compared to charging the filling stations for a filling operation.

The plant is operated in a make-to-order manner. Orders specify the quantity of a demanded final (packaged) product. In most cases, the demands exceed the maximum processing capacity of the units in the plant and therefore the orders have to be separated into batches.

The formulation stage consists of seven parallel processing lines, where each formulation line is equipped with a mixing tank that blends liquids and powders, and an intermediate buffer tank that feeds a mill. The mixing tank is repeatedly charged with raw materials and in between these charging operations mixing takes place. After the milling step, the intermediate products are supplied to two parallel ripening tanks, where a final ingredient is dosed and a minimum ripening time has to elapse. From the ripening tanks, the intermediate products may either be stored in one of the available buffer tanks or the intermediates are directly transferred to the filling stage. When neither a buffer tank nor a filling station is available for storage or further processing, the intermediates can also remain inside one of the ripening tanks prior to discharging. In addition to these formulation lines, theres one special formulation 
line, denoted "Black Box line". Its operation is not modeled in detail and this formulation line produces a different kind of intermediate. Beyond that, this "Black Box line" interactions with the buffer tanks and the filling stations in the same as for the other formulation lines.

Full equipment connectivity is given, i.e. each formulation line may feed each buffer tank and filling station. In the filling stations, the intermediates are packaged into a variety of final containers. In contrast to the formulation lines, the filling stations work in a semicontinuous manner, i.e. when a filling operation takes place, the source tank (a buffer tank or a ripening tank) is gradually discharged during the filling time, necessitating synchronous operation of the source equipment and the filling equipment. There is no nightshift, and thus the filling stations are idle at night, which requires intermediates to be stored in the buffer tanks or the ripening tanks overnight. Operations on the filling stations can be interrupted during the night and resumed the next day. In case of operator shortage preemption is possible as well.

All charging and discharging operations are performed with constant flowrates. Since multiple batches of formulated intermediate can be fed to the buffer tanks, the buffer tanks may reach their upper capacity limit. In this case, the charging operation from the formulation tank is interrupted until the buffer tank has discharged enough of its content to a filling station. The intermediate tanks can be simultaneously charged and discharged.

In all of the equipment, sequence-dependent changeover times have to elapse (usually for cleaning) if two subsequent batches, storage operations or filling operations use different raw materials or intermediate products.

Within the scope of this work, the processing times of all operations are deterministic, but product- and line-dependent. In addition, it is assumed that the orders are known a priori as well. Each order has a due date, that applies to the finishing time of the last filling operation of the last batch for this order.

Two objectives are investigated in this case study: completion time and tardiness. With $i \in \mathcal{I}$ being an order in the set of orders, $j \in \mathcal{J}$ being an unit in the set of units and $C_{i, j}$ being the finishing time of the last operation that is processed on unit $j$ and belongs to order $i$, the completion time is defined as:

$$
C T=\sum_{j \in \mathcal{J}} C_{j}
$$

with

$$
C_{j}=\max _{i \in \mathcal{I}}\left\{C_{i, j}\right\} .
$$

The total tardiness is defined as:

$$
T=\sum_{i \in \mathcal{I}} \max \left(0, C_{i}-d_{i}\right),
$$

where $d_{i}$ is the due date and $C_{i}$ is the finishing time of the last operation of order $i$.

\section{SCHEDULING APPROACH}

Figure 2 shows the proposed approach. We encode the scheduling decisions in two distinct chromosomes, an allocation and a sequence chromosome which are modified by genetic operations and inherited independently. In the initialization step, the two chromosomes, which are introduced in detail in Subsection 3.2, are initialized according to a predefined initialization strategy, which is explained in Subsection 3.5. For the fitness evaluation, the encoded scheduling decisions are implemented by the DES during the simulation. The DES then returns the objective function value, i.e. either the completion time or the tardiness, and whether the schedule is feasible. Details on the feasibility of the genotypes are given in Subsection 3.6. Detailed discussions on the design of the survivor and parent selection and the genetic operators are provided in Subsections 3.4 and 3.3, respectively.

\subsection{Search space heuristics}

As mentioned above the investigated case study is too complex for including all available degrees of freedom in the optimization. This requires the systematic use of heuristics to obtain a search space of reasonable size that is expected to still contain the optimum or at least high-quality solutions close to the optimum. This is a critical step in the design of the optimization approach. First the available degrees of freedom are outlined. The complete list of available degrees of freedom is:

(1) Allocation: For each batch of each order, it can be decided on which formulation lines, intermediate buffer tanks (if one is used) and filling stations the batches are processed or stored. Within the formulation line, an operation that takes place in the equipment of one line cannot be routed to be processed on equipment of another line for the subsequent operation. Therefore, once a batch is processed on the mixing tank of a formulation line it needs to stay on that line until discharging to a buffer tank or a filling station takes place. In addition, the decision in which of the two parallel formulation tanks a batch is ripened is part of the allocation decisions.

(2) Sequencing: On each formulation line and on each filling station, the sequence of the batches that are processed must be chosen. A batch that started in the mixing tank cannot be overtaken by another batch until the batches reached the parallel ripening tanks. In these tanks, the intermediate storage time is not limited, a batch $B$ that has been started later in the mixing tank than a batch $A$ can leave the formulation line earlier than batch $A$. In the filling stations and buffer tanks, batches can be sequenced arbitrarily, but may not be mixed between products.

(3) Routing: It must be decided if a batch is stored in one of the buffer tanks at all, or if the intermediates are directly routed from one of the ripening storage tanks to one of the filling stations.

(4) Batching: An order in most cases consists of multiple batches. The number of batches per order are fixed by the customer demand of the product in question. The batch sizes are assumed to be a plant-wide constant. Every batch can have a unique allocation, sequence and route through the plant.

(5) Timing: The timing decisions include the start time of a batch and idle times that can be introduced at almost arbitrary positions between operations. Idle times can be beneficial for example if a batch should overtake another batch, to achieve a better changeover time. In the DES this can be enforced only by introducing idle times or interrupting operations intentionally. 


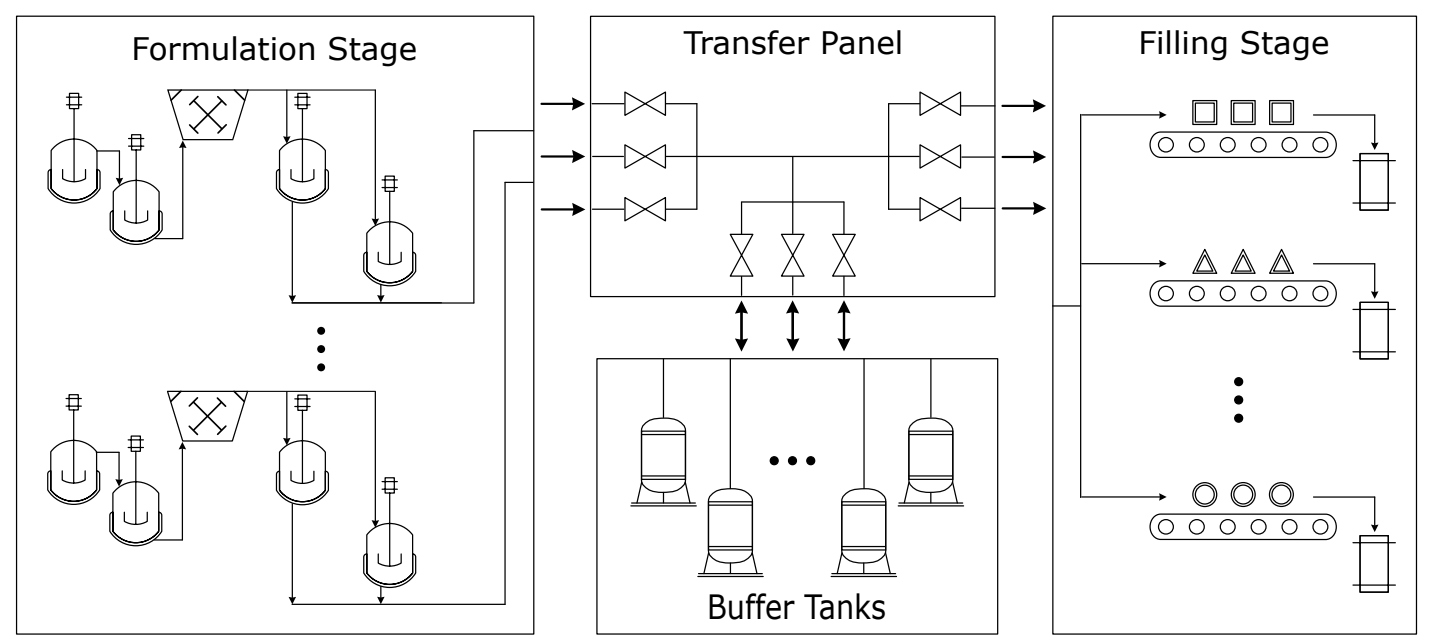

Figure 1: Schematic representation of the processing plant.

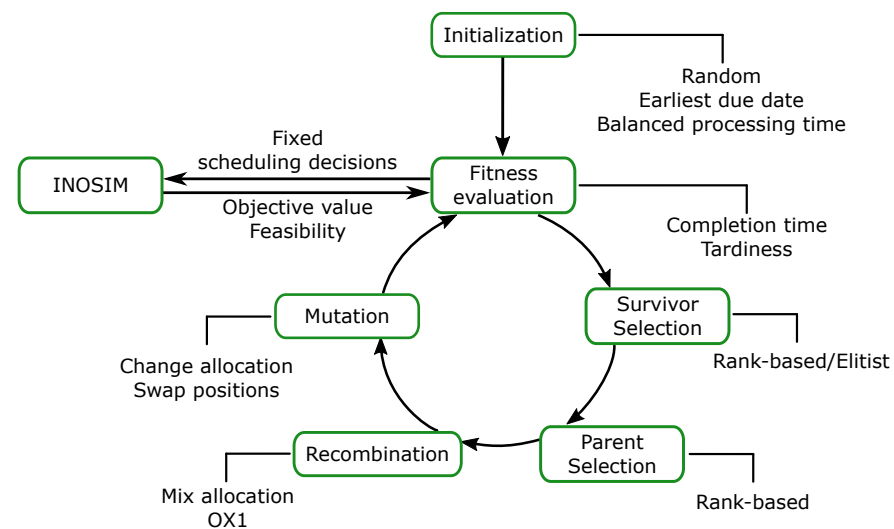

Figure 2: Schematic representation of the simulation-based Genetic Algorithm.

\subsection{Encodings}

This section deals with the encoding of the production schedules.

The formulation stage and the filling stage consist of several nonidentical parallel lines. The non-identity of the processing lines lies in the differences in processing time that is needed to formulate a product or to fill a product into its final container. Because of the significant differences in the processing times, the allocation of orders to a formulation line has a large impact on the quality of a solution. Therefore we choose a direct encoding of the allocation, which is illustrated in Figure 3. In this example orders 1, 4 and 5 are allocated to Line 3, while no order is allocated to Line 4.

We employ an order-wise allocation encoding in contrast to a batch-wise encoding. This reduces the size of the allocation chromosome and thus the search space. Also, the changeover times are smaller when batches belonging to the same order are scheduled on the same unit and follow each other. In this paper we do not consider order splitting.

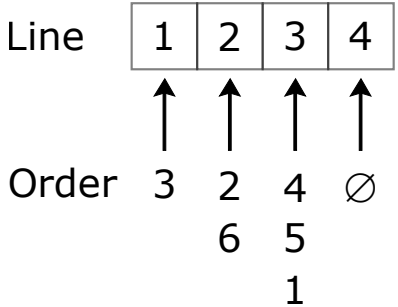

Figure 3: Schematic representation of the allocation encoding.

Secondly, we encode the sequence of orders globally. This leads to a high degree of coupling between the formulation stage and the filling stage, despite the fact that in principle the sequence in the filling stage could be completely decoupled from the formulation sequence through the use of the buffer tanks.

A decoding procedure is designed for the DES, such that it can apply the scheduling decisions, that are defined by the allocation and sequence chromosome, in the simulation. The global sequence is directly applied to the formulation lines for the given unit allocation. However, for the filling stations, the determination of the sequences in the simulation is more involved. Primarily, an earliest start time heuristic determines the sequence. This means, that a batch is filled into the filling stations as soon as it is available, if the target filling station is free. If the target filling station is unavailable and a buffer tank is free, the intermediate is stored in a free buffer tank. A buffer tank can be charged if one of the following conditions applies: it is either empty or an identical intermediate product is currently stored, and the buffer tank is currently not supplied by another formulation line. Intermediates from a buffer tank that compete with intermediates that come directly from the formulation lines are given lower priority.

Since a simple, yet reasonable routing heuristic exists, that decides whether a batch passes the buffer tanks, and since the expected 
impact of the routing decision is expected to be small, we do not encode the routing as a degree of freedom.

We also renounce from encoding the timing degrees of freedom. Except for rare cases, finishing the operations as early as possible is a locally optimal decision. With respect to the infinite size of the search space of timing decisions and the additional effort that is necessary to realize intentional interruption or delays of operations in the DES, the determination of the timing is performed solely by the DES.

The production plant under consideration bears a structural similarity to a flexible flowshop. Out of the encodings proposed for flexible flowshop problems (see [14] for a thorough comparison of different representations), none suits our needs perfectly. Although in [14] it was shown by means of an example, that simpler representations where only a job sequence is encoded and the assignment is determined in a rule-based fashion can yield better results when given the same computational time, we decided to encode the allocation directly as well. The reason for this is that the proposed optimization framework should be flexible and is applicable to other scheduling problems.

\subsection{Mutation and Recombination}

For both types of chromosomes, i.e. the allocation and the sequence chromosome, a mutation and a recombination operator have been designed. Recombination is always applied to obtain a child from two parent individuals. For the allocation chromosome a custom recombination operator is employed. The allocation chromosome recombination operator iterates over all orders of the chromosome and randomly selects either the allocation from the first or from the second parent (Mix allocation in Figure 2). For the sequence chromosome, we apply the well-known OX1 crossover operator as introduced in [4].

Mutation is applied with a probability of $p_{M u t}$. For the allocation chromosome, a random order is chosen and a new (not the same) line is sampled from the set of eligible lines with equal probability, where this order is allocated (Change allocation in Figure 2). For the sequence chromosome the positions of two random orders in the sequence are swapped (Swap positions in Figure 2).

\subsection{Parent and survivor selection}

For the survivor selection, we use a $(\mu+\lambda)$ selection, where $\mu$ denotes the size of the population and $\lambda$ denotes the number of offspring generated per generation [1]. The selection operator is the rank-based roulette wheel selection, as described in [7]. Compared to the standard roulette wheel selection, it has the advantage that a set of solutions with similar objective function values can still be assigned dissimilar probabilities of selection. This is especially useful in situations, where the spread between the high- and lowquality solutions in a population is small. This selection scheme uses a selective pressure parameter $1 \leq S P_{\text {survivor }} \leq 2$. A high value of $S P$ gives more weight to high-quality solutions, whereas with $S P_{\text {survivor }}=1$ all solutions are selected with equal probability. It was observed that in many cases promising solutions do not survive for more than a few generations, even when setting $S P_{\text {survivor }}=2$. To better exploit the traits of these solutions in recombination, it is desirable that these solutions survive as long as no better solutions are found. Hence, we extend the survivor selection procedure partly by an elitist selection, the fittest $p_{\text {elitist }} * \mu$ individuals are selected with certainty and from the remaining $\left(1-p_{\text {elitist }}\right) * \mu$ individuals, the survivors are selected according to the rank-based roulette wheel selection.

The parent selection only uses the rank-based roulette wheel selection. The corresponding tuning parameter is denoted $S P_{\text {parent }}$.

\subsection{Initialization strategy}

For each chromosome, a heuristic initialization procedure is proposed in addition to random initialization.

For the allocation chromosome, we use a procedure that balances the total allocated processing time across the formulation lines. In this initialization strategy, first a random sequence of all orders is generated. The orders are then assigned sequentially to the processing line with the least accumulated total processing time. This strategy is referred to as the Balanced Processing Time (BPT) heuristic. Ties are broken randomly. The procedure is inspired by the Random permutation method proposed in [2].

For the sequence chromosome, arbitrary static dispatching rules (i.e. rules based on properties that are known prior to simulation) can be used for initialization. In our case, the Earliest Due Date (EDD) dispatching rule is used for the tardiness objective. This means that all orders are sorted in ascending order according to their due date, where ties are broken randomly. For the completion time objective, we use a random initialization. Beyond that, our encoding guarantees that the batches belonging to a single order are scheduled en bloc which leads to short changeover times.

\subsection{Constraint handling}

Constraint handling is an issue that needs special treatment in the realm of evolutionary algorithms [3]. In our case almost all constraints such as synchronization, timing, precedence, changeover, resource limitations (personnel) are handled by the DES in the sense that the DES produces an executable schedule from the two chromosomes. The only exceptions are deadlocks that can arise, when too many orders are forced to be processed on the same formulation line or filling station by an inappropriate choice of the allocation. In these cases, a constant, large penalty is added to the objective function.

\section{RESULTS}

The results section is structured as follows: First an exemplary solution for a representative case that involves two weeks of production is presented. Then the results of the proposed optimization approach are benchmarked against the schedule generation by the DES, simple heuristics and randomly generated solutions. Finally, the reproducibility of the solutions is verified, showing that the repeated execution of the algorithm leads to solutions of comparable quality.

The exemplary case for which the results are shown in this section comprises 17 orders of three different products that necessitate the production of 72 batches. In this example, a tight coupling between intermediate and final products exists, i.e. there are only three final products, that are produced from three intermediate products. 
Table 1: Parameters of the Genetic Algorithm

\begin{tabular}{ccl}
\hline Parameter & Symbol & Value \\
\hline Population size & $\mu$ & 20 \\
Number of children & $\lambda$ & 20 \\
Number of generations & $n_{G e n}$ & 50 \\
Mutation rate & $p_{M u t}$ & 0.3 \\
Parent selection pressure parameter & $S P_{\text {parent }}$ & 1.5 \\
Survivor selection pressure parameter & $S P_{\text {survivor }}$ & 1.85 \\
Fraction of the elite & pelitist & 0.4 \\
\hline
\end{tabular}

Due to the detailed simulation the single evaluation with the DES takes approximately $40 \mathrm{~s}$ (from the start of the simulation until the results are written). Therefore, several simulations are executed in parallel, up to 20 simulation instances can be solved concurrently.

The optimizations were conducted on a FUJITSU PRIMERGY RX300 S8 with two 12-core Intel Xeon E5-2697 processors $(2.70 \mathrm{GHz})$ and 192 GB of RAM. The optimizer was implemented in C\#7.3 on the .NET framework Version 4.6.1. As DES INOSIM 13.0 Runtime Edition, provided by the company Inosim Software $\mathrm{GmbH}$, was used.

Since the GA was implemented as a generational GA, the optimizer waits for all instances to return the simulation result until it proceeds with the survivor selection. However, the time needed for a single simulation to finish differs depending on the specific scheduling decisions that are simulated. Therefore the net speedup is significantly smaller than 20 since the GA has to wait for the slowest simulation in each generation.

Table 1 depicts the parameter that were used to generate the results. To satisfy computational time constraints implied by the end users, quick convergence is desirable. Therefore we set $S P_{\text {survivor }}$ to 1.85 and $p_{\text {elitist }}$ to 0.4 . Similarly we set $S P_{\text {parent }}$ to 1.5 , slightly higher than reported in [12] for the survivor selection, to favor the selection of well-performing parents even more. We set the mutation rate to $p_{M u t}=0.3$. Beyond that, we ran preliminary experiments where the mutation rate $p_{M u t}$ was varied, but we could not observe a significant impact of this parameter. Only 1020 evaluations of schedules are possible $\left(n_{\text {Gen }} \cdot \lambda+\mu\right)$ within the computational time constraints of about $2 \mathrm{~h}$. An implication of the current parallelized implementation is that $\mu$ and $\lambda$ should be equal and should be chosen such that the computational resources are fully utilized. We chose $\mu=\lambda=20$, because this has shown to be the smallest value, where the total capacity of the processors is utilised.

In Figure 4, an exemplary schedule that was obtained as the best-known solution of a minimization of the tardiness is displayed. The formulation lines are denoted "FL", the mixing and formulation buffer tanks are indexed with "B01" and "B02", the mills are indexed with "Mill", and the ripening tanks are indexed with "B03" and "B04". The "Black Box line" is represented only by a single piece of equipment, which is denoted "FL8_BlackBox". The filling stations are denoted "FS" and the intermediate buffer tanks are denoted "ST". "FS3" represents the third filling station, and "ST6" represents the sixth intermediate storage tank.
Table 2: Due dates and number of batches for each order.

\begin{tabular}{lcc}
\hline \multicolumn{1}{c}{ Order } & $\begin{array}{c}\text { Due Date } \\
\text { [dd.mm.yyyy }]\end{array}$ & $\begin{array}{c}\text { Batches per } \\
\text { order }\end{array}$ \\
\hline $\begin{array}{l}10 X X 00 \text { where } X \in \\
\{01,04,07,10,13,16\}\end{array}$ & 08.01 .2020 & 2 \\
$10 X X 00$ where $X \in$ & 12.01 .2020 & 4 \\
$\{02,05,08,11,14,17\}$ & \\
$\begin{array}{l}10 X X 00 \text { where } X \in \\
\{03,06,09,12,15\}\end{array}$ & 04.01 .2020 & 7 \\
\hline
\end{tabular}

Each distinct horizontal colored block represents a single operation, e.g. charging, discharging, cleaning, waiting or processing. Groups of identically colored blocks represent operations that belong to the same order. The numbers inside horizontal blocks indicate the order that an operation belongs to. The order numbers of filling operations make it possible to trace them back to the corresponding formulation order. For example "11020000" belongs to formulation order "100200". The light green vertical blocks indicate the nightshifts; operations which take place during these time periods are interrupted during the night and resumed the next morning. The due dates imposed on the orders are given in Table 2. The starting date of the schedule is 01.01.2020 12 AM.

Operations in the mixing tanks "B01", formulation buffer tanks "B02" and the mills are allocated at the same time, which is a consequence of the DES simulation. However, only transfer operations between the equipment takes place synchronously in the real plant, whereas all other processing operations take place independently.

In the Gantt chart, orders with early due dates have higher priority compared to orders with later due dates in most formulation and filling stations. The only exception among the orders with a due date at 04.01 .2020 is order " 100600 ", which contributes the most to the value of the tardiness for this schedule. One expects that in a high-quality solution, order "100600" is the first order produced on either "FL2", "FL4" or "FL6", while here orders with later due dates are produced first. However, the processing, charging and discharging rates depend on the line and the type of product produced. Processing order "100600" would take considerably longer on "FL2", "FL4" or "FL6" such that "FL7" is a reasonable choice. Solutions where "100600" was swapped with "100400" on line "FL6" or "101300" on line "FL2" were tested explicitly and lead to worse tardiness values.

Another observation from the Gantt chart is that the intermediate buffer tanks are used in almost all cases. These units are utilized even more than the filling units because the routing heuristic that is implemented prefers to use the intermediate buffer over a direct discharging into the filling units from the ripening tanks. As mentioned in Section 2, direct discharging from the formulation tanks into the filling units is a time-consuming task. It is usually beneficial to charge finished intermediates from the ripening tanks into the intermediate buffer tanks to free up the capacity in the formulation units for the subsequent batches.

This exemplary Gantt chart also reveals where the improvement potential for future work lies. In this work, a complete order that 


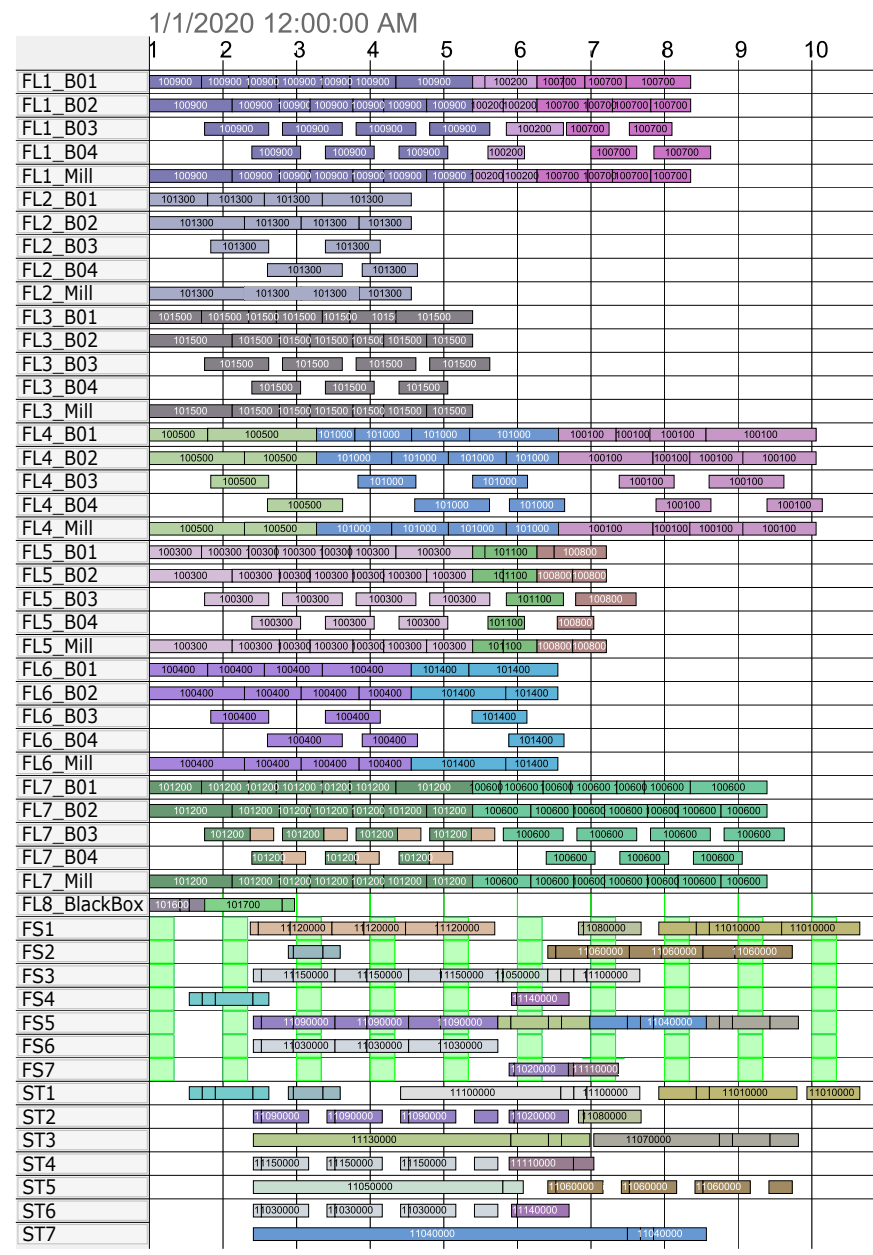

Figure 4: Exemplary schedule of the case study with 72 batches.

may consist of several batches was represented by a single element in both the allocation and sequence chromosomes. This was done to reduce the search space of the optimization problem to a tractable size. Nevertheless, from the Gantt chart it is deducible that the solutions can be improved by splitting the orders into smaller sub-orders and allocate them to different lines to process them in parallel.

In Table 3, the objective function values of the solutions obtained from the optimization approach are given. For both objectives, tardiness and completion time, the performance of the optimization approach ("optimized") is compared to the solutions that the DES yields when applied without optimization ("default"), to randomly generated chromosomes ("random") and to heuristically generated chromosomes ("EDD"/"BPT"). Since both the allocation and sequence chromosomes are decoded by the DES independently different combinations of the different approaches can be implemented.

The proposed optimization approach outperforms all other approaches significantly, even for a random initialization. Randomly
Table 3: Results of the optimization and the default results of the DES for completion time and tardiness.

\begin{tabular}{cccl}
\hline $\begin{array}{c}\text { Approach } \\
\text { allocation }\end{array}$ & $\begin{array}{c}\text { Approach } \\
\text { sequence }\end{array}$ & Objective & Value $[\mathrm{h}]$ \\
\hline Optimized & Optimized & Completion time & $6706^{*}$ \\
Random & Random & Completion time & $7630^{* *}$ \\
Default & Default & Completion time & 8706 \\
Optimized & Optimized & Tardiness & $312^{*}$ \\
Random & Random & Tardiness & $351^{* *}$ \\
BPT & EDD & Tardiness & $428^{* *}$ \\
Random & EDD & Tardiness & $569^{* *}$ \\
Default & Default & Tardiness & 610 \\
Default & EDD & Tardiness & 740 \\
\hline
\end{tabular}

* Best objective function values averaged over 5 optimization runs.

** Best objective function value of 1000

randomly generated chromosomes.

generated chromosomes perform well in comparison with the default solution. The comparatively poor performance of the DES default solutions has several reasons. First, the DES without the optimizer is not aware of due dates and the tardiness objective at all and can therefore not take decisions to specifically reduce the tardiness. Secondly, the DES simulates the schedule in a manner that is similar to a First Come First Serve heuristic. To put it simply, the DES traverses all event points in ascending order and whenever a unit becomes idle, it picks the next operation from the queue and dispatches it on the unit. Since the processing rates of the units depend on the line and type of product, this heuristic takes suboptimal decisions frequently. A user that wants to minimize the tardiness would consider the due dates when entering the list or orders by choosing a permutation of the orders, where orders with early due dates will mostly occur in early positions. In this case, the sequence of the list of orders that was used in the default case, was provided by the industrial case study provider and was not previously optimized.

For the completion time objective, the EDD and BPT heuristics do not lead to reasonable results, which is why they have not been included. For EDD this is straightforward. Balancing the loads of the units is not a promising strategy to minimize the completion time, since allocating the units with shorter processing times leads to a smaller sum of all processing times, which is highly correlated with the completion time. It is rather beneficial to utilize the units with lower processing times as much as possible and leave the units with high processing times idle, which contradicts the idea of line balancing.

Since a GA is non-deterministic, the reproducibility of the performance of the proposed approach was validated via repeated execution of the optimization. Figures 5 and 6 illustrate the reproducibility of five runs with completion time and tardiness as objectives. In both figures, the mean and the minimum objective function value of the population in each generation is displayed. The instance that was solved is identical to the one that was used 


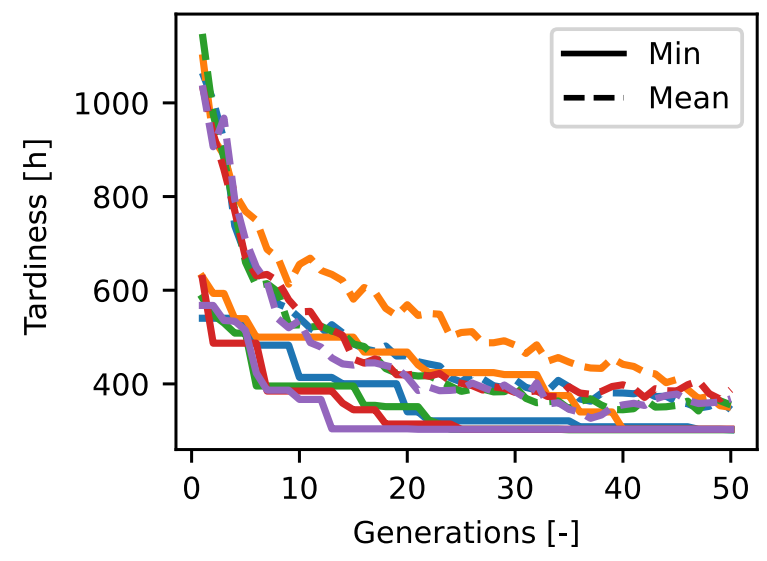

Figure 5: Reproducibility plot for the tardiness optimization of 5 runs of the GA.

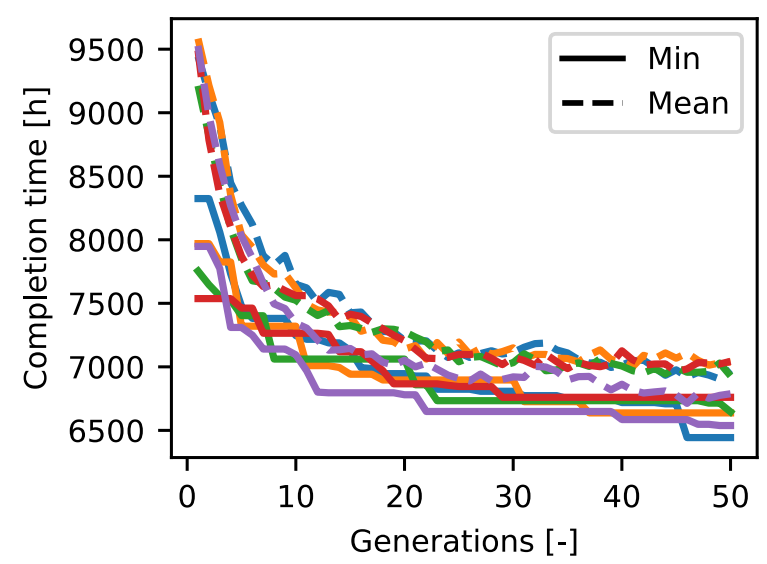

Figure 6: Reproducibility plot for the completion time optimization of 5 runs of the GA.

for the exemplary schedule. A random initialization was chosen for these optimization runs.

For the optimization of the tardiness the GA reliably converges to solutions close to $300 \mathrm{~h}$. In the reproducibility plot of the completion time optimization, the best solutions start in the range of $\sim 7500 \mathrm{~h}$ up to $\sim 8320 \mathrm{~h}$ in the first generation and converge to solutions in the range $\sim 6760 \mathrm{~h}$ to $\sim 6440 \mathrm{~h}$, with a standard deviation of $119 \mathrm{~h}$. Keeping in mind the complexity of this industrial case study, a coefficient of variation of $\sim 1.8 \%$ for the completion time is acceptable.

\section{SUMMARY, CONCLUSION AND OUTLOOK}

In this work we propose an optimization approach to solve a scheduling problem for a real-world industrial two-stage formulation and filling batch production plant. Sequence-dependent changeover times, unit and product-dependent processing times, limited intermediate storage, a semi-continuous filling stage, operator shift constraints and transfer operation synchronization are amongst the features of this scheduling problem.
The complex processing characteristics are captured by a detailed discrete event simulator (DES) model. The DES is used as a schedule builder and evaluation module by the genetic algorithm. Special attention has been paid to the encoding of the degrees of freedom. Different heuristics are employed in order to trade off the growth of the search space versus the expected impact on the solution quality when deciding how a certain degree of freedom is encoded. Some degrees of freedom, such as the allocation decisions for the intermediate buffer tanks, have therefore not been encoded at all but have been left to be determined by the DES during the simulation via a predefined dispatching rule.

We could demonstrate that the optimization approach yields schedules where reasonable scheduling decisions have been taken when considering the due dates of the orders and the varying processing rates of the units. Furthermore we showed, that the approach yields reproducible results of high-quality, which far outperform randomly generated solutions, simple heuristics and the default simulation results of the DES.

For reasons of higher flexibility, we also decided to decouple the encoding of the sequence and the allocation as much as possible. When applying the proposed approach to another problem, one can easily exchange the encoding of the allocation or the sequence degrees of freedom, but does not have to come up with an encoding for the remaining degrees of freedom.

A future challenge that we will tackle is to incorporate the batching decisions into the encoding. Especially with respect to the tardiness optimization, an improvement in solution quality can be expected. We intend to use an additional chromosome to encode how an order is split into sub-orders. This has direct implications for the allocation and sequencing chromosome. In these two chromosomes, the number of elements to sequence or to allocate will change, which renders the design of the algorithm more difficult.

Another open issue is the interaction of the sequence and the allocation chromosomes. The sequence chromosome has as many elements as there are orders. The number of permutations for the sequence chromosome is larger than the number of different sequences that can be realized with a given allocation. The mutation operator may swap two orders in the sequence that run on different processing lines, but the resulting phenotype can be identical to the one before mutation. Therefore we aim to implement allocationaware operators that act on the sequence chromosome in crossover and mutation.

Finally, a thorough parameter tuning and testing of different recombination and mutation operators will be conducted to improve the optimization approach.

\section{ACKNOWLEDGMENTS}

This work was partially funded by the European Regional Development Fund (ERDF) in the context of the project OptiProd.NRW (https://www.optiprod.nrw/en).

We would like to thank Vassilios Yfantis for providing feedback on the manuscript.

\section{REFERENCES}

[1] Hans-Georg Beyer and Hans-Paul Schwefel. 2002. Evolution strategies - A comprehensive introduction. Natural Computing 1 (2002), 3 - 52. https://doi. org/10.1023/A 
[2] Tsung-che Chiang and Hsiao-jou Lin. 2013. Int . J . Production Economics A simple and effective evolutionary algorithm for multiobjective flexible job shop scheduling. Intern. Journal of Production Economics 141, 1 (2013), 87-98. https://doi.org/10.1016/j.ijpe.2012.03.034

[3] Carlos A Coello Coello. 2002. Theoretical and Numerical Constraint-Handling Techniques used with Evolutionary Algorithms: A Survey of the State of the Art Computer Methods in Applied Mechanics and Engineering 191, 11-12 (2002), 12451287. https://doi.org/10.1017/CBO9781107415324.004 arXiv:arXiv:1011.1669v3

[4] L Davis. 1985. Applying Adaptive Algorithms to Epistatic Domains. Proc. of IfCAI-85 (1985), 162-164.

[5] Shaohua Dong and D. J. Medeiros. 2012. Minimising schedule cost via simulation optimisation: An application in pipe manufacturing. International fournal of Production Research 50, 3 (2012), 831-841. https://doi.org/10.1080/00207543.2010. 545447

[6] Frédéric Dugardin, Farouk Yalaoui, and Lionel Amodeo. 2010. New multiobjective method to solve reentrant hybrid flow shop scheduling problem. $\mathrm{Eu}$ ropean fournal of Operational Research 203, 1 (2010), 22-31. https://doi.org/10. 1016/j.ejor.2009.06.031

[7] A.E. Eiben and J.E. Smith. 2015. Introduction to Evolutionary Computing. SpringerVerlag Berlin Heidelberg. 287 pages. https://doi.org/10.1108/03684920410699216

[8] Iiro Harjunkoski, Christos T. Maravelias, Peter Bongers, Pedro M. Castro, Sebastian Engell, Ignacio E. Grossmann, John Hooker, Carlos Méndez, Guido Sand, and
John Wassick. 2014. Scope for industrial applications of production scheduling models and solution methods. Computers and Chemical Engineering 62 (2014), 161-193. https://doi.org/10.1016/j.compchemeng.2013.12.001

[9] D. Istokovic, M. Perinic, M. Vlatkovic, and M. Brezocnik. [n.d.]. Minimizing Total Production Cost in a Hybrid Flow Shop: a Simulation-Optimization Approach. 19, 4 ([n. d.]), 559-570. https://doi.org/10.2507/IJSIMM19-4-525

[10] A. Oyarbide-Zubillaga, A. Goti, and A. Sanchez. [n.d.]. Preventive maintenance optimisation of multi-equipment manufacturing systems by combining discrete event simulation and multi-objective evolutionary algorithms. 19, 4 ([n.d.]), 342-355. https://doi.org/10.1080/09537280802034091

[11] Sabine Piana and Sebastian Engell. 2010. Hybrid evolutionary optimization of the operation of pipeless plants. Fournal of Heuristics 16, 3 (2010), 311-336. https://doi.org/10.1007/s10732-009-9105-7

[12] Noraini Mohd Razali and John Geraghty. 2011. Genetic algorithm performance with different selection strategiesin solving TSP. Proceedings of the World Congress on Engineering 2011, WCE 20112 (2011), 1134-1139.

[13] F. Rossi, P. van Beek, and T. Walsh. 2006. Handbook of Constraint Programming. Elsevier B.V., Amsterdam.

[14] Thijs Urlings, Ruben Ruiz, and Funda Sivrikaya Serifoglu. 2010. Genetic algorithms with different representation schemes for complex hybrid flexible flow line problems. International fournal of Metaheuristics 1, 1 (2010), 30. https://doi.org/10.1504/ijmheur.2010.033122 